Please do not remove this page

RMIT

UNIVERSITY

\title{
The transmitted and reflected waves due to the incidence of a forced wave on a junction
}

Davy, John; Irwin, Alexandra

https://researchrepository.rmit.edu.au/esploro/outputs/9921860349001341/filesAndLinks?institution=61RMIT_INST\&index=null

Davy, J., \& Irwin, A. (2016). The transmitted and reflected waves due to the incidence of a forced wave on a junction. Building Acoustics, 23(1), 3-16. https://doi.org/10.1177/1351010X15627008

Document Version: Accepted Manuscript

Published Version: https://doi.org/10.1177/1351010X15627008

Repository homepage: https://researchrepository.rmit.edu.au

(c) The Author(s) 2016

Downloaded On 2023/04/26 14:16:15 +1000

Please do not remove this page 
Thank you for downloading this document from the RMIT Research Repository.

The RMIT Research Repository is an open access database showcasing the research outputs of RMIT University researchers.

RMIT Research Repository: http://researchbank.rmit.edu.au/

\section{Citation:}

Davy, J and Irwin, A 2016, 'The transmitted and reflected waves due to the incidence of a forced wave on a junction', Building Acoustics, vol. 23, no. 1, pp. 3-16.

See this record in the RMIT Research Repository at:

https://researchbank.rmit.edu.au/view/rmit:36169

Version: Accepted Manuscript

Copyright Statement: (C) The Author(s) 2016

Link to Published Version:

http://dx.doi.org/10.1177/1351010X15627008 


\title{
The Transmitted and Reflected Waves due to the Incidence of a Forced Wave on a
}

\section{Junction}

\author{
John L. Davy ${ }^{1,2}$, Alexandra L. Irwin ${ }^{1}$ \\ ${ }^{1}$ School of Applied Sciences, Royal Melbourne Institute of Technology (RMIT) University, \\ GPO Box 2476V Melbourne, Victoria 3001, Australia, john.davy@rmit.edu.au \\ ${ }^{2}$ CSIRO Materials Science and Engineering, Private Bag 10, Clayton, Victoria 3168, \\ Australia,john.davy@csiro.au
}

\begin{abstract}
The airborne sound transmission through a plate below its critical frequency is dominated by forced waves even though the plate vibration is dominated by freely propagating waves. This paper studies whether the transmission of the two wave types is different at a junction. It is shown in this paper that it is not possible to separately calculate in a meaningful way the intensity of a forced incidence wave and its freely propagating reflected wave in the medium from which incidence occurs because the cross terms in the intensity calculation for the combined waves, which vanish when the incident wave is freely propagating, do not vanish when the incident wave is forced. This means that it is not possible to define a transmission factor for forced incident waves. Thus the transmitted intensity must be calculated as a function of the mean square wave amplitude of the forced wave.
\end{abstract}

\section{INTRODUCTION}

When finite sized plate is excited into transverse vibration by an airborne sound field incident on one side of the plate, two types of bending wave field are produced in the plate. The first is the forced bending wave field which is directly excited by the incident airborne sound field. The wave length of the forced bending wave field is determined by the wavelength and angle of incidence of the exciting airborne sound field. This is the only bending wave field which would exist if the plate was infinite in extent. For a finite size plate, the forced bending wave field is reflected at the edges of the plate and the reflected waves are the freely propagating bending wave field. The wavelength of the freely propagating bending wave field is determined by the bending wave phase speed of the plate and the frequency of the freely propagating bending wave field. In terms of the partial differential equation for bending waves on a plate, the forced bending wave field is the particular integral which is a solution of the non-homogeneous bending wave equation which is forced by the sound pressure exerted on the plate by the incident airborne sound field. The freely propagating bending wave field is the solution of homogeneous bending wave equation 
with no forcing term which together with the forced bending wave field satisfies the bending wave boundary conditions of the plate.

Below the critical frequency of a panel, the panel's airborne sound insulation is dominated by the forced waves in the panel, even though the panel's vibration is dominated by the freely propagating waves. This is because the radiation efficiency of the forced waves is much greater than the radiation efficiency of the freely propagating waves below the critical frequency. This difference in radiation efficiency has led to the theory of airborne flanking sound transmission considering the two types of wave separately below the critical frequencies of the panels. Above the critical frequency it is not experimentally possible to distinguish between the two types of wave.

Gerretsen ${ }^{1}$, in his first paper on flanking sound transmission, stated that "For a flanking transmission path only free vibrations should be considered below the critical frequency". This will be correct if the bending wave intensity transmitted at a junction between panels by freely propagating waves is much greater than that transmitted by the forced waves. This will be the case if the amplitude of the freely propagating waves is much greater than the amplitude of the forced waves providing the transmission of the forced waves is not much greater than the transmission of the freely propagating waves at the panel junction. Alternatively it will also be the case if the transmission of the freely propagating waves at the panel junction is much greater than the transmission of the forced waves providing the amplitude of the forced waves is not much greater than the amplitude of the freely propagating waves. The first situation will usually apply, but Villot and Guigou-Carter ${ }^{2}$ showed experimentally, using near field acoustical holography, that near the mass air mass resonant frequency of a lightweight cavity wall, the amplitude of the forced waves dominated the amplitude of the freely propagating waves.

Irwin $^{3}$, under the supervision of Davy, has researched the intensity transmitted at junctions by incident forced waves for the case of a junction between acoustic half infinite media and for the case of a pinned junction between two half infinite plates. Of particular interest was the situation of forced incident diffuse fields, and in the plate case the situation when one of the plates is forced by a diffuse sound field. The purpose of this paper is to make Irwin's results available in the international refereed learned journal literature

\section{THE ACOUSTIC CASE}

In the plate case, the energy is transported by the interaction of the transverse velocity with the transverse force and by the interaction of the moment with the angular velocity. The situation is simpler in the acoustic medium case because the energy is transported only by the 
interaction of the acoustic pressure with the acoustic particle velocity. For this reason, Irwin ${ }^{3}$ studied the acoustic case first before proceeding to the more complicated case of the junction between two plates. While it is probably not possible to practically generate a three dimensional forced sound field, a one dimensional forced sound field is produced inside a microphone turbulence screen and a two dimensional forced sound field is produced in the cavity of a double wall, providing the cross sectional dimension of both is very small compared to the wave length of the sound.

The case of two half infinite acoustic media which are joined at the infinite plane interface $x=0$ is considered in this section. The geometry of the situation considered is shown in Figure 1. The medium 1 occupies the space $x<0$ and medium 2 occupies the space $x>0$. The media have freely propagating wave numbers $k_{1}$ and $k_{2}$ and characteristic impedances $Z_{1}$ and $Z_{2}$, respectively. A forced plane wave with acoustic pressure root mean square (rms) amplitude $p_{i}$ is incident from medium 1 upon the plane interface with wave number $\mathbf{k}_{i}=\left(k_{i x}, 0, k_{i z}\right)=\left(k_{i} \cos \left(\theta_{i}\right), 0, k_{i} \sin \left(\theta_{i}\right)\right)$ where $\theta_{i}$ is the angle of incidence of the forced wave number relative to the positive $x$-axis and $k_{i}=\left|\mathbf{k}_{i}\right|$. It should be noted that there is no loss of generality by assuming that the $y$ - axis component $k_{i y}$ of $\mathbf{k}_{i}$ is zero. The Cartesian co-ordinate system axes can always be rotated about the $x$ - axis to zero the $y$-axis component of the incident forced wave number.

The incidence of the forced incident wave on the interface produces a freely propagating reflected wave in medium 1 and a freely propagating transmitted wave in medium 2. The transmitted wave has acoustic pressure rms amplitude $t p_{i}$ with wave number $\mathbf{k}_{2}=\left(k_{2 x}, 0, k_{2 z}\right)$ and $k_{2}=\left|\mathbf{k}_{2}\right|$. The reflected wave has acoustic pressure rms amplitude $r p_{i}$ with wave number $\mathbf{k}_{1}=\left(-k_{1 x}, 0, k_{1 z}\right)$ and $k_{1}=\left|\mathbf{k}_{1}\right|$.

Define

$$
\begin{gathered}
\chi_{a}=\frac{k_{i}}{k_{1}}, \\
\kappa=\frac{k_{2}}{k_{1}}, \\
\beta=\frac{Z_{2}}{Z_{1}}, \\
s^{2}=\chi_{a}^{2} \sin ^{2}\left(\theta_{i}\right) .
\end{gathered}
$$


In order to satisfy the continuity conditions at the interface $(x=0)$, the reflected and transmitted waves must have the same component of wave number in the positive $z$-axis direction as the incident wave, namely

$$
k_{2 z}=k_{1 z}=k_{i z}=k_{i} \sin \left(\theta_{i}\right),
$$

where 2, 1 and $i$ denote the transmitted, reflected and incident waves.

The reflected wave has a wave number component in the negative $x$-axis direction with magnitude $k_{x 1}$ given by

$$
k_{1 x}=k_{1} \sqrt{1-\chi_{a}^{2} \sin ^{2}\left(\theta_{i}\right)} .
$$

Note that this is only a propagating wave if $\chi_{a}\left|\sin \left(\theta_{i}\right)\right| \leq 1$. If $\chi_{a}\left|\sin \left(\theta_{i}\right)\right|>1$, this reflected wave becomes a non-propagating near field wave. If the reflected wave is a propagating wave, its direction of propagation makes an angle of $\theta_{1}$ with the negative $x$-axis direction given by

$$
\left|\sin \left(\theta_{1}\right)\right|=\chi_{a}\left|\sin \left(\theta_{i}\right)\right|
$$

The transmitted wave has a wave number component in the positive $x$-axis direction with magnitude $k_{x 2}$ given by

$$
k_{2 x}=k_{1} \sqrt{\kappa^{2}-\chi_{a}^{2} \sin ^{2}\left(\theta_{i}\right)} .
$$

Note that this is only a propagating wave if $\chi_{a}\left|\sin \left(\theta_{i}\right)\right| \leq \kappa$. If $\chi_{a}\left|\sin \left(\theta_{i}\right)\right|>\kappa$, this transmitted wave becomes a non-propagating near field wave. If the transmitted wave is a propagating wave, its direction of propagation makes an angle of $\theta_{2}$ with the positive $x$-axis direction given by

$$
\left|\sin \left(\theta_{2}\right)\right|=\frac{\chi_{a}}{\kappa}\left|\sin \left(\theta_{i}\right)\right|
$$

Applying the continuity conditions at the interface $(x=0)$ gives

$$
\begin{array}{r}
t=\frac{\beta \kappa\left(\sqrt{\chi_{a}^{2}-s^{2}}+\sqrt{1-s^{2}}\right)}{\beta \kappa \sqrt{1-s^{2}}+\sqrt{\kappa^{2}-s^{2}}}, \\
r=\frac{\beta \kappa \sqrt{\chi_{a}^{2}-s^{2}}-\sqrt{\kappa^{2}-s^{2}}}{\beta \kappa \sqrt{1-s^{2}}+\sqrt{\kappa^{2}-s^{2}}} .
\end{array}
$$

The intensity $I_{t x}$ transmitted in the positive $x$ - axis direction from medium 1 into medium 2 at the media interface $(x=0)$ is given by the following equation. 


$$
\frac{2 Z_{2} I_{t x}}{\left|p_{i}\right|^{2}}=\frac{2 \beta^{2} \kappa\left|\left(\sqrt{\chi_{a}^{2}-s^{2}}+\sqrt{1-s^{2}}\right)\right|^{2} \operatorname{Re}\left(\sqrt{\kappa^{2}-s^{2}}\right)}{\left|\beta \kappa \sqrt{1-s^{2}}+\sqrt{\kappa^{2}-s^{2}}\right|^{2}} .
$$

For an incident diffuse sound field, the lower limit of integration can be set equal to

$$
g_{l}= \begin{cases}\sqrt{1-\frac{\kappa^{2}}{\chi_{a}^{2}}} \text { if } \chi_{a}>\kappa \\ 0 \quad \text { if } \chi_{a} \leq \kappa\end{cases}
$$

because of the occurrence of total internal reflection. The integral over the hemisphere of all possible solid angles of incidence uses a $\sin \left(\theta_{i}\right)$ weighting factor because the hemisphere has more area as a function of incident angle close to grazing angles of incidence. The substitution $g=\cos \left(\theta_{i}\right)$ is used.

The intensity $<I_{t x}>$ transmitted in the positive $x$ - axis direction from medium 1 into medium 2 at the media interface $(x=0)$ by an incident forced diffuse sound field is given by the following equation.

$$
\frac{2 Z_{2}\left\langle I_{t x}\right\rangle}{\left|p_{i}\right|^{2}}=2 \int_{g_{l}}^{1} \frac{\beta^{2} \kappa\left|\left(\chi_{a} g+\sqrt{1-\chi_{a}^{2}+\chi_{a}^{2} g^{2}}\right)\right|^{2} \operatorname{Re}\left(\sqrt{\kappa^{2}-\chi_{a}^{2}+\chi_{a}^{2} g^{2}}\right)}{\left|\beta \kappa \sqrt{1-\chi_{a}^{2}+\chi_{a}^{2} g^{2}}+\sqrt{\kappa^{2}-\chi_{a}^{2}+\chi_{a}^{2} g^{2}}\right|^{2}} \mathrm{~d} g .
$$

The integral in eqn (14) usually has to be numerically evaluated. However, if $\kappa=1$, the integral can be analytically evaluated to give the following very simple equation.

$$
\frac{|1+\beta|^{2} Z_{2}\left\langle I_{t x}\right\rangle}{2|\beta|^{2}\left|p_{i}\right|^{2}}=\left\{\begin{array}{ll}
\left(1+\chi_{a}\right) / 2 & \text { if } \chi_{a} \leq 1 \\
\left(1+1 / \chi_{a}\right) / 2 & \text { if } \chi_{a} \geq 1
\end{array} .\right.
$$

Note that if $\beta=1$, the normalization of the left hand side of eqn (15) is the same as the normalization of the left hand sides of eqns (12) and (14). However, when eqn (14) was graphed by $\operatorname{Irwin}^{3}$, it was normalized by dividing it by its value when $\chi_{a}=1$.

The incident intensity $I_{(i+r) x}$ in the positive $x$-axis direction on the media interface $(x=$ 0 ) due to the combined incident and reflected waves can be shown, as must be the case, to be equal to the transmitted intensity $I_{t x}$. This incident intensity is not equal to the sum of the intensity of the incident wave $I_{i x}$ in the positive $x$-axis direction on the media interface $(x=0)$ and the intensity of the reflected wave $I_{r x}$ in the positive x-axis direction on the media interface $(x=0)$ unless the incident wave is freely propagating. This can be shown easily by considering the normally incident case $\left(\theta_{i}=0\right)$ when $\chi_{a}=1$ and $\beta=1$. There is still an interface at $x=0$ because the forcing function is only non-zero when $x<0$. 
The intensities for this demonstration are normalized by dividing by the intensity $I_{i x 1}$ of the incident wave if it was freely propagating. The intensity of $I_{i x}$ of the incident wave in the absence of the reflected wave is

$$
\frac{I_{i x}}{I_{i x 1}}=\chi_{a}
$$

The intensity $I_{r x}$ of the reflected wave in the absence of the incident wave is

$$
\frac{I_{r x}}{I_{i x 1}}=-\left(\frac{1-\chi_{a}}{2}\right)^{2}=-\left(\frac{\chi_{a}-1}{2}\right)^{2} .
$$

Thus the sum of the incident and reflected wave intensities in the positive $x$ - axis direction is

$$
\frac{I_{i x}+I_{r x}}{I_{i x 1}}=\frac{-\chi_{a}^{2}+6 \chi_{a}-1}{4}
$$

The intensity due to the combined incident and reflected waves and the transmitted intensity is given by

$$
\frac{I_{(i+r) x}}{I_{i x 1}}=\frac{I_{t x}}{I_{i x 1}}=\frac{\chi_{a}^{2}+2 \chi_{a}+1}{4} .
$$

Note that eqns (18) and (19) are not equal unless $\chi_{a}=1$. In other words they are only equal if the incident wave is freely propagating.

The main purpose of this section has been to show that it is not possible to calculate the net intensity which is incident on the junction by vectorially summing the intensities of the incident and reflected waves when the incident wave is forced. The reason for this is that the cross terms between the incident and reflected waves in the sound intensity calculation for the combined wave do not cancel unless the incident wave is freely propagating. This means that it is not possible to meaningfully calculate the incident intensity of the incident wave on its own, and hence that it is not possible to meaningfully calculate the transmission factor, which is the ratio of the transmitted to the incident intensity, unless the incident wave is freely propagating.

It can also be seen, without the above mathematics, that this must be true by considering the case of an incident forced wave which has the same instantaneous values everywhere in the incident medium. Such a forced wave would be produced in a wall cavity by an external plane sound wave which is incident normally on one of the leaves of the wall cavity. This incident forced wave has a wave number of zero, and thus has zero intensity. Yet, at the junction between the two media where the forcing function stops acting, a freely 
propagating reflected wave and a freely propagating transmitted wave will be produced because of the amplitude continuity conditions at the forcing function discontinuity. Thus there is a forced incident wave with zero intensity and freely propagating reflected and transmitted waves which on their own can only carry energy away from the forcing function discontinuity. This produces the paradox that energy is being transported away from the forcing function discontinuity while no energy is being injected at the forcing function discontinuity. This paradox disappears if the intensity of the combination of the forced incident wave and the freely propagating reflected wave is calculated.

It should also be noted that the forcing function can inject energy into or remove energy from the forced incident wave in any region of the medium in which the incident wave is being forced. This means that the intensity incident on a junction due to the presence of a forced incident wave, and the reflected wave that the incidence of the forced wave produces, can only be calculated at the junction.

\section{TRANSMISSION OF BENDING WAVES AT A PINNED JOINT}

In this section, the bending wave intensity transmitted at the pinned line junction of two rigidly connected infinite half plates, when a forced bending wave is incident on the pinned line junction, is calculated. The geometry of the situation considered is shown in Figure 1. The pinned line junction is chosen, because the situation is equivalent to two infinite half plates rigidly connected at right angles to each other along an infinite line junction, if longitudinal waves are ignored. Each infinite half plate restricts the transverse motion of the other infinite half plate, connected at right angles to the first plate, to be zero at the infinite line junction, if the longitudinal waves are ignored. Because the two plates are at right angles and rigidly connected, the infinite line junction is held linearly but not rotationally motionless. Making the infinite line junction a pinned junction achieves the same result regardless of the angle between the plates. It stops any motion of the junction by providing any needed force, but allows the transmission of bending wave power from one plate to the other plate by the interaction of angular velocities and moments. Pinning the infinite line junction stops the generation of the longitudinal waves which are being ignored in the right angle connection case. Thus in this section, the two plates are assumed to lie in the same plane without any loss of generality.

The plates are inline in the $y=0$ plane. Plate 1 is the infinite half plane given by $y=0$ and $x \leq 0$. Plate 2 is the infinite half plane given by $y=0$ and $x \geq 0$. The two plates are rigidly joined along the line $x=0$ and $y=0$. The incident and reflected bending waves are in plate 1 
and the transmitted bending waves are in plate 2. The ratios of the root mean square (rms) amplitudes of the propagating reflected wave, the near field reflected wave, the propagating transmitted wave and the near field transmitted wave to the amplitude of the forced incident wave, are $r, r_{j}, t, t_{j}$ respectively. $k_{1}$ and $k_{2}$ are the freely bending propagating wave numbers of plates 1 and 2 respectively. The forced incident wave has a forced bending wave number $k_{i}$ and is incident at an angle of $\theta_{i}$ relative to the positive $x$-axis which is the normal to the junction line between the two plates.

The rms transverse velocities of plates 1 and 2 , normalized by dividing by the rms transverse velocity of the incident wave are

$$
\begin{gathered}
{\left[\exp \left(-j k_{i x} x\right)+r \exp \left(j k_{1 x} x\right)+r_{j} \exp \left(k_{1 N} x\right)\right] \exp \left(-k_{1 z} z\right) \exp (j \omega \tau) \text { if } x \leq 0,} \\
{\left[t \exp \left(-j k_{2 x} x\right)+t_{j} \exp \left(-k_{2 N} x\right)\right] \exp \left(-k_{2 z} z\right) \exp (j \omega \tau) \text { if } x \geq 0,}
\end{gathered}
$$

where $\omega$ is the angular frequency and $\tau$ is the time.

The magnitude of the component of the incident bending wave number in the $z$-axis direction is

$$
k_{i z}=k_{i} \sin \left(\theta_{i}\right) .
$$

The propagating reflected wave has a bending wave number component in the negative $x$-axis direction with magnitude $k_{x 1}$ given by

$$
k_{1 x}=k_{1} \sqrt{1-\chi_{a}^{2} \sin ^{2}\left(\theta_{i}\right)} .
$$

Note that this is only a propagating wave if $\chi_{a}\left|\sin \left(\theta_{i}\right)\right|<1$. If $\chi_{a}\left|\sin \left(\theta_{i}\right)\right|>1$, this reflected wave becomes a non-propagating near field wave. If the reflected wave is a propagating wave, its direction of propagation makes an angle of $\theta_{1}$ with the negative $x$-axis direction given by

$$
\left|\sin \left(\theta_{1}\right)\right|=\chi_{a}\left|\sin \left(\theta_{i}\right)\right|
$$

The reflected decaying near field has a bending wave number component in the negative $x$-axis direction with magnitude $k_{N 1}$ given by

$$
k_{1 N}=k_{1} \sqrt{1+\chi_{a}^{2} \sin ^{2}\left(\theta_{i}\right)} .
$$

The propagating transmitted wave has a bending wave number component in the $x$-axis direction with magnitude $k_{x 2}$ given by

$$
k_{2 x}=k_{1} \sqrt{\kappa^{2}-\chi_{a}^{2} \sin ^{2}\left(\theta_{i}\right)}
$$


Note that this is only a propagating wave if $\chi_{a}\left|\sin \left(\theta_{i}\right)\right|<\kappa$. If $\chi_{a}\left|\sin \left(\theta_{i}\right)\right|>\kappa$, this transmitted wave becomes a non-propagating near field wave. If the transmitted wave is a propagating wave, its direction of propagation makes an angle of $\theta_{2}$ with the positive $x$-axis direction given by

$$
\left|\sin \left(\theta_{2}\right)\right|=\frac{\chi_{a}}{\kappa}\left|\sin \left(\theta_{i}\right)\right|
$$

The transmitted decaying near field has a bending wave number component in the positive $x$-axis direction with magnitude $k_{N 2}$ given by

$$
k_{2 N}=k_{1} \sqrt{\kappa^{2}+\chi_{a}^{2} \sin ^{2}\left(\theta_{i}\right)} .
$$

For a freely propagating incident wave $\left(\chi_{a}=1\right)$, eqns $(23),(25),(26)$ and (28) agree with eqns $(6.142 \mathrm{a})$ to $(6.142 \mathrm{~d})$ of Cremer et al. ${ }^{4}$.

Define

$$
\psi=\frac{B_{2}}{B_{1}} \kappa^{2}=\frac{B_{2} k_{2}^{2}}{B_{1} k_{1}^{2}},
$$

where $B_{1}$ and $B_{2}$ are the bending stiffnesses of plates 1 and 2 respectively.

Define

$$
\Delta=\psi\left(j \sqrt{1-s^{2}}-\sqrt{1+s^{2}}\right)+\left(j \sqrt{\kappa^{2}-s^{2}}-\sqrt{\kappa^{2}+s^{2}}\right) .
$$

The ratios of the root mean square (rms) amplitudes of the propagating transmitted wave, the propagating reflected wave, the near field transmitted wave, and the near field reflected wave, to the rms amplitude of the forced incident waves, are

$$
\begin{gathered}
t=\frac{\left(j \sqrt{\chi_{a}^{2}-s^{2}}+\sqrt{1+s^{2}}\right)+\left(j \sqrt{1-s^{2}}-\sqrt{1+s^{2}}\right)\left(\chi_{a}^{2}+1\right) / 2}{\Delta}, \\
r=\frac{\psi\left(j \sqrt{\chi_{a}^{2}-s^{2}}+\sqrt{1+s^{2}}\right)-\left(j \sqrt{\kappa^{2}-s^{2}}-\sqrt{\kappa^{2}+s^{2}}\right)\left(\chi_{a}^{2}+1\right) / 2}{\Delta}, \\
t_{j}=-\frac{\left(j \sqrt{\chi_{a}^{2}-s^{2}}+\sqrt{1+s^{2}}\right)+\left(j \sqrt{1-s^{2}}-\sqrt{1+s^{2}}\right)\left(\chi_{a}^{2}+1\right) / 2}{\Delta}, \\
r_{j}=\frac{-j \psi\left(\sqrt{\chi_{a}^{2}-s^{2}}+\sqrt{1-s^{2}}\right)+\left(j \sqrt{\kappa^{2}-s^{2}}-\sqrt{\kappa^{2}+s^{2}}\right)\left(\chi_{a}^{2}-1\right) / 2}{\Delta} .
\end{gathered}
$$

For a freely propagating incident wave $\left(\chi_{a}=1\right)$, eqns (31) and (32) agree with eqns (6.147a) and (6.147b) of Cremer et al. ${ }^{4}$. 
The transmitted bending wave power $\left(I_{t x}\right)$ in the direction of the positive $x$-axis per unit length of the plate junction is given by the following equation.

$$
\frac{I_{t x}}{2 m_{2} c_{2}\left|v_{1+}\right|^{2}}=|t|^{2} \operatorname{Re}\left(\sqrt{1-\frac{s^{2}}{\kappa^{2}}}\right),
$$

where $m_{2}$ and $c_{2}$ are the mass per unit area and the bending wave speed respectively of plate 2 and $\left|v_{1+}\right|$ is the root mean square velocity of the forced incident bending wave.

The transmitted bending wave power $(I)$ per unit length of the plate junction is only nonzero when

$$
\chi_{a}^{2} \sin ^{2}\left(\theta_{i}\right)=s^{2}<\kappa^{2}
$$

This gives

$$
\theta_{i}<\arcsin \left(\frac{\kappa}{\chi_{a}}\right)
$$

Define the upper limit of integration as

$$
\theta_{u}=\left\{\begin{array}{ll}
\frac{\pi}{2} & \text { if } \kappa \geq \chi_{a} \\
\arcsin \left(\frac{\kappa}{\chi_{a}}\right) & \text { if } \kappa<\chi_{a}
\end{array} .\right.
$$

The power per unit length $\left\langle I_{t x}\right\rangle$ transmitted to plate 2 by a forced diffuse bending wave field in plate 1 is given by

$$
\frac{\pi\left\langle I_{t x}\right\rangle}{2 m_{2} c_{2}|v|^{2}}=\int_{0}^{\theta_{u}}|t|^{2} \operatorname{Re}\left(\sqrt{1-\frac{s^{2}}{\kappa^{2}}}\right) \mathrm{d} \theta_{i} .
$$

where $|v|$ is the root mean square diffuse transverse velocity in plate 1 .

If the transverse bending vibration in plate 1 is forced on one of its sides by a plane sound wave, with wave number $k_{a}$ in the fluid medium, travelling at an angle of $\phi$ to the normal to plate 1 , the wave number $k_{i}$ of the forced wave in plate 1 is (the geometry of the situation considered is shown in Figure 1 and Figure 2)

$$
k_{i}=k_{a} \sin (\phi),
$$

and

$$
\begin{gathered}
\chi_{a}=\frac{k_{i}}{k_{1}}=\frac{k_{a} \sin (\phi)}{k_{1}}, \\
s^{2}=\chi_{a}^{2} \sin ^{2}\left(\theta_{i}\right)=\frac{k_{a}^{2} \sin ^{2}(\phi) \sin ^{2}\left(\theta_{i}\right)}{k_{1}^{2}} .
\end{gathered}
$$


Eqn (41) is the reason for the use of the subscript $a$ on the variable $\chi_{a}$.

The modulus squared of the wave impedance of plate 1 is

$$
\left|Z_{p}\right|^{2}=\frac{\omega^{2}}{\left\{\left[k_{a}^{4} \sin ^{4}(\phi)-k_{1}^{4}\right]^{2}+\eta^{2} k_{a}^{8} \sin ^{4}(\phi)\right\} B_{1}^{2}},
$$

where $\eta$ is the damping loss factor of panel 1 .

The power per unit length $\left\langle I_{t x}\right\rangle_{a}$ transmitted to plate 2 by a bending wave field in plate 1 , which is forced by an incident diffuse sound field in the fluid medium on one side of plate 1 , is given by

$$
\frac{\pi B_{1}^{2}\left\langle I_{t x}\right\rangle_{a}}{m_{2} c_{2} \omega^{2}|p|^{2}}=\int_{0}^{\pi / 2} \int_{0}^{\theta_{u}} \frac{|t|^{2} \operatorname{Re}\left(\sqrt{1-\frac{s^{2}}{\kappa^{2}}}\right) \sin (\phi)}{\left[k_{a}^{4} \sin ^{4}(\phi)-k_{1}^{4}\right]^{2}+\eta^{2} k_{a}^{8} \sin ^{4}(\phi)} \mathrm{d} \theta_{i} \mathrm{~d} \phi,
$$

where $|p|$ is the root mean square diffuse sound pressure.

Define

$$
q=\frac{k_{a}}{k_{1}}=\sqrt{\frac{\omega}{\omega_{c 1}}}=\sqrt{\frac{f}{f_{c 1}}},
$$

where $\omega$ and $f$ are the angular frequency and the frequency and $\omega_{c i}$ and $f_{c i}$ are the critical angular frequency and the critical frequency of plate $i$. The angular critical frequency and the critical frequency are the angular frequency and frequency respectively when the sound wave number $k_{a}$ in the fluid medium equals the bending wave number $k_{i}$ in the plate. It should also be noted that

$$
\kappa=\frac{k_{2}}{k_{1}}=\sqrt{\frac{\omega_{c 2}}{\omega_{c 1}}}=\sqrt{\frac{f_{c 2}}{f_{c 1}}} .
$$

Figure 3 shows the transmitted intensity in plate 2 of a bending wave, forced by an incident diffuse acoustic sound field on one side of plate 1 in $\mathrm{dB}$ as a function of the ratio $q$ of the wave number of the diffuse sound field to the freely propagating bending wave number in plate 1 . The mean square sound pressure of the incident diffuse sound field is kept constant as $q$ varies since it is not possible to meaningfully calculate the incident intensity for a forced incident wave. Plate 2 is assumed to have the same material properties as plate 1 and the infinite length joint is assumed to be pinned. The curve of Davy \& Irwin is the right hand side of eqn (44) converted to decibels and normalized so that it is equal to $0 \mathrm{~dB}$ when $q=1$. The curve of Davy \& Irwin uses a damping loss factor of $\eta=0.03$ because Villot and GuigouCarter $^{2}$ performed their calculations for two plates of $10 \mathrm{~mm}$ thick gypsum plaster board. 
The second author's thesis ${ }^{3}$ contains very detailed derivations of the equations presented in this paper. It also contains a large number of graphs of the equations presented in this paper. This thesis is available for download from RMIT University's research repository at http://researchbank.rmit.edu.au/eserv/rmit:160444/Irwin.pdf .Thus, in the interests of brevity, these derivations and graphs have not been included in this paper, with the exception of the very important Figure 3.

\section{COMPARISON WITH THE RESULTS OF VILLOT AND GUIGOU-CARTER}

Because some of the results given in this paper differ from those given by Villot and Guigou-Carter ${ }^{2}$, this section examines the differences and the reasons for those differences. Eqn (31) agrees with eqn (9) of Villot and Guigou-Carter ${ }^{2}$ apart from differing in sign. This difference in sign is due to the fact that the two plates are in the same plane in this paper, while in Villot and Guigou-Carter ${ }^{2}$ the second plate has been rotated through 90 degrees in order to form a right angled junction. Thus the velocity in the positive $y$-axis direction in the second plate becomes a velocity in the negative $x$-axis direction in the Villot and GuigouCarter $^{2}$ paper. Note that the positive direction of the $x$-axis in figure $6 \mathrm{a}$ of Villot and GuigouCarter $^{2}$ is incorrect. It does not correspond to the signs of the exponential exponents in their eqn (4). Also note that once the positive direction of the $x$-axis is changed in their figure 6 , the positive direction of the $z$-axis also has to be changed in order to maintain a right handed co-ordinate system.

The first error in the paper of Villot and Guigou-Carter ${ }^{2}$ is that they divide eqn (35) by the intensity of the forced incident wave on its own in order to obtain the transmission efficiency in their eqn (10). As shown in section 2, the calculation of the intensity of the forced incident wave on its own does not produce sensible results unless the incident wave is freely propagating. It is easy to see that eqn (10) of Villot and Guigou-Carter ${ }^{2}$ does not always produce sensible results. A normally incident plane sound wave forces a transverse velocity wave with zero wave number and infinite phase speed in plate 1 . The zero wave number gives zero stand-alone incident intensity [see eqn (3.92) of Cremer et al. ${ }^{4}$ ] and hence infinite transmission efficiency using the approach of Villot and Guigou-Carter ${ }^{2}$. Examination of eqns (35) and (31) and energy conservation show that this cannot be correct. Eqn (10) of Villot and Guigou-Carter ${ }^{2}$ actually predicts zero transmission efficiency in this case because the equation for calculating bending wave intensity used by Villot and GuigouCarter $^{2}$ actually assumes that the incident bending wave is freely propagating, which is not valid for an incident forced bending wave. 
It should be noted that Villot and Guigou-Carter ${ }^{2}$ did not include the damping loss factor that occurs in eqns (43) and (44).

The second error occurs in eqn (13) of Villot and Guigou-Carter ${ }^{2}$ which calculates the transmission factor when the incident plate is excited on one side by an incident airborne diffuse sound field. Expanding Villot and Guigou-Carter's weighting functions in their eqn (13) produces a weighting function which contains the cosine of the incident angle of the incident airborne diffuse acoustic sound field which excites the incident plate. This cosine term should not be in the weighting function because the amplitude of the forced wave in the panel depends on the amplitude rather than the intensity on the surface of the plate of the incident airborne diffuse acoustic sound field.

The integration in eqn (44) uses an angular weighting factor of $\sin (\phi)$ in order to account for the fact that there more solid angle for angles of incidence close to grazing incidence. This angular weighting is different from that used in eqn (13) of Villot and Guigou-Carter ${ }^{2}$. In the notation of this paper, Villot and Guigou-Carter evaluate an integral of the form

$$
\int_{0}^{k_{a}} \int_{0}^{\pi / 2} F\left[\theta_{i}, k_{i}\right] k_{i} \mathrm{~d} \theta_{i} \mathrm{~d} k_{i} .
$$

Since

$$
k_{i}=k_{a} \sin (\phi), \quad \mathrm{d} k_{i}=k_{a} \cos (\phi) \mathrm{d} \phi,
$$

Villot and Guigou-Carter's integral becomes

$$
\int_{0}^{\pi / 2} \int_{0}^{\pi / 2} F\left[\theta_{i}, k_{a} \sin (\phi)\right] k_{a}^{2} \sin (\phi) \cos (\phi) \mathrm{d} \theta_{i} \mathrm{~d} k_{i} .
$$

The extra $\cos (\phi)$ factor occurs when the incident sound wave intensity on a surface is being calculated. However, in this case, the rms amplitude of the forced transverse vibrational bending wave in plate 1 depends on the rms amplitude of the incident sound wave and not on the incident intensity of the sound wave on plate 1. Thus Villot and Guigou-Carter's second error is the use of the $\cos (\phi)$ factor.

Figure 3 compares the calculations graphed in figure 7 of Villot and Guigou-Carter ${ }^{2}$ with those made in this paper. The curve of Villot and Guigou-Carter is inverted to give transmission rather than transmission loss and normalized using their resonant transmission value from their figure 7.

The values of $q$ for the curve in figure 7 of Villot and Guigou-Carter ${ }^{2}$ are calculated by taking the square root of the ratio of the frequency divided by the critical frequency. In figure 
7 of Villot and Guigou-Carter ${ }^{2}$, the critical frequency is where their forced curve stops decreasing with increasing frequency and becomes almost constant above $3.4 \mathrm{kHz}$

The freely propagating bending wave case is approximately $0 \mathrm{~dB}$ in Figure 3 . The freely propagating case is also the $q \geq 1$ case in Figure 3 . This is because when $q \geq 1$, the freely propagating transverse velocity waves generated by coincidence dominate the vibration of plate 1. Coincidence occurs when the component of the acoustic wave number parallel to the surface is equal to the freely propagating transverse wave number of plate 1 . When $q \geq 1$, the Davy \& Irwin curve and the Villot \& Guigou-Carter curve are both close to $0 \mathrm{~dB}$.

For values of $q \geq 1$, the integral in eqn (44) is dominated by values of $\chi_{a}$ close to one. In this case

$$
k_{a}|\sin (\phi)| \approx k_{1} \text {. }
$$

Because the damping loss factor $\eta$ is small (0.03), the denominator of the integrand in eqn (44) is close to zero when eqn (50) applies. This means that the integrand has a sharp maximum at $\chi_{a}=1$. The effect is that the integral is relatively constant for $q \geq 1$. Because the integral is normalized by dividing by its value when $q=1$, the normalized integral is close to one which is $0 \mathrm{~dB}$ when $q \geq 1$.

The critical frequency occurs when $q=1$. Above the critical frequency, where $q \geq 1$, the two curves in Figure 3 agree well with each other. Below the critical frequency, the relative transmitted intensity calculated in this paper is much greater than that calculated by Villot and Guigou-Carter ${ }^{2}$. Hence it is not as obvious as claimed by Villot and Guigou-Carter 2 that the intensity transmitted by the forced waves can be ignored relative to that transmitted by resonant waves. The production of Figure 3 was the main aim of this paper. The identification of two errors in Villot and Guigou-Carter's ${ }^{2}$ calculations meant that it was important to correct their calculations. This has been achieved in Figure 3 and large differences between the corrected calculations of this paper and Villot and Guigou-Carter's ${ }^{2}$ calculations have been identified below the critical frequency.

\section{CONCLUSION}

This paper derived the transmitted intensity due to forced incident waves at a junction for both the acoustic case and the case of two plates connected at a pinned junction It has shown that the intensity incident on an interface cannot be calculated as the difference of the intensity of the incident wave minus the intensity of the reflected wave unless the incident wave is freely propagating. The reason is that the cross terms which appear in the calculation of the intensity of the combined wave do not cancel unless the incident wave is freely 
propagating. This means that it is not possible to define a transmission factor for forced waves as Villot and Guigou ${ }^{2}$ attempted to do.

This paper has also shown that there are two errors in the derivation of Villot and Guigou-Carter ${ }^{2}$. These two errors have been shown to lead to Villot and Guigou-Carter ${ }^{2}$ under estimating the transmission of forced bending waves relative to the transmission of freely propagating bending waves at a pinned junction of plates below the critical frequency of the plates. This means that it is not as obvious as Villot and Guigou-Carter have suggested that "the transmission of forced vibration can be neglected" at a junction of plates.

\section{References}

1. Gerretsen, E., Calculation of the sound-transmission between dwellings by partitions and flanking structures. Applied Acoustics, 1979, 12(6), 413-433.

2. Villot, M. and Guigou-Carter, C. Contribution of forced and resonant vibration in sound transmission through partitions and in vibration transmission through plate junctions, in: NOVEM 2000, Lyon, France, 2000 of Conference, 1-10.

3. Irwin, A.L., The transmission of forced waves at a junction, Master of Applied Science Thesis, RMIT University, 2013.

4. Cremer, L., Heckl, M., and Petersson, B.A.T., Structure-Borne Sound - Structural Vibrations and Sound Radiation at Audio Frequencies, 3rd edn, Springer, Berlin, Germany, 2005. 


\section{Figures}

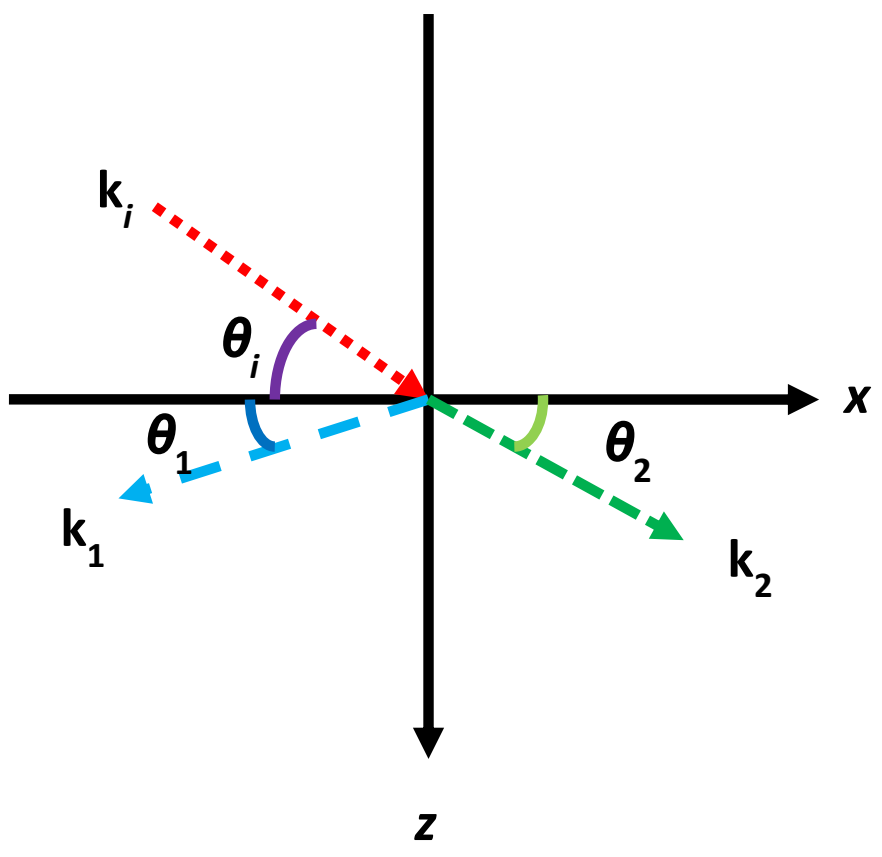

Figure 1 . The geometry of the situation considered in this paper. The $y$-axis points vertically out of the page. Medium 1 (or plate 1) occupies the volume $x \leq 0$ (or the area $x \leq 0, y=0$ ) and medium 2 (or plate 2) occupies the volume $x \geq 0$ (or the area $x \geq 0, y=0$ ). 


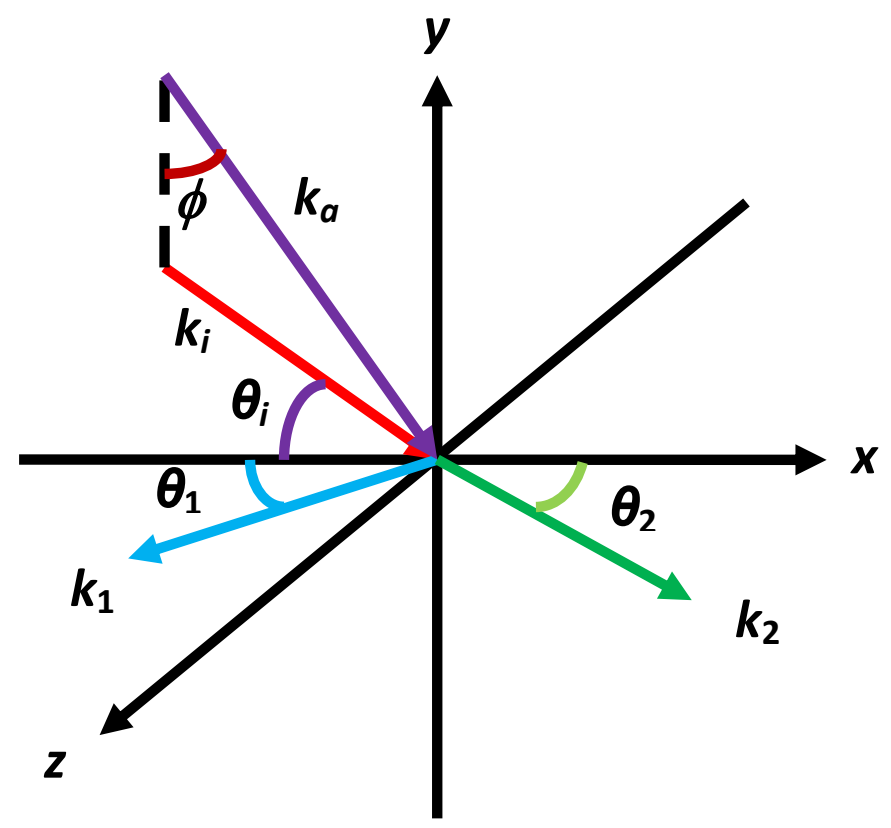

Figure 2. The geometry of the situation when plate 1 is excited on one side by a plane sound wave. Plate 1 occupies the area $x \leq 0, y=0$ and plate 2 occupies the area $x \geq 0, y=0$.The geometry in the plane $y=0$ is shown in Figure 1 . The wave number vectors $\mathbf{k}_{i}, \mathbf{k}_{1}$ and $\mathbf{k}_{2}$ lie entirely in the plane $y=0$. 


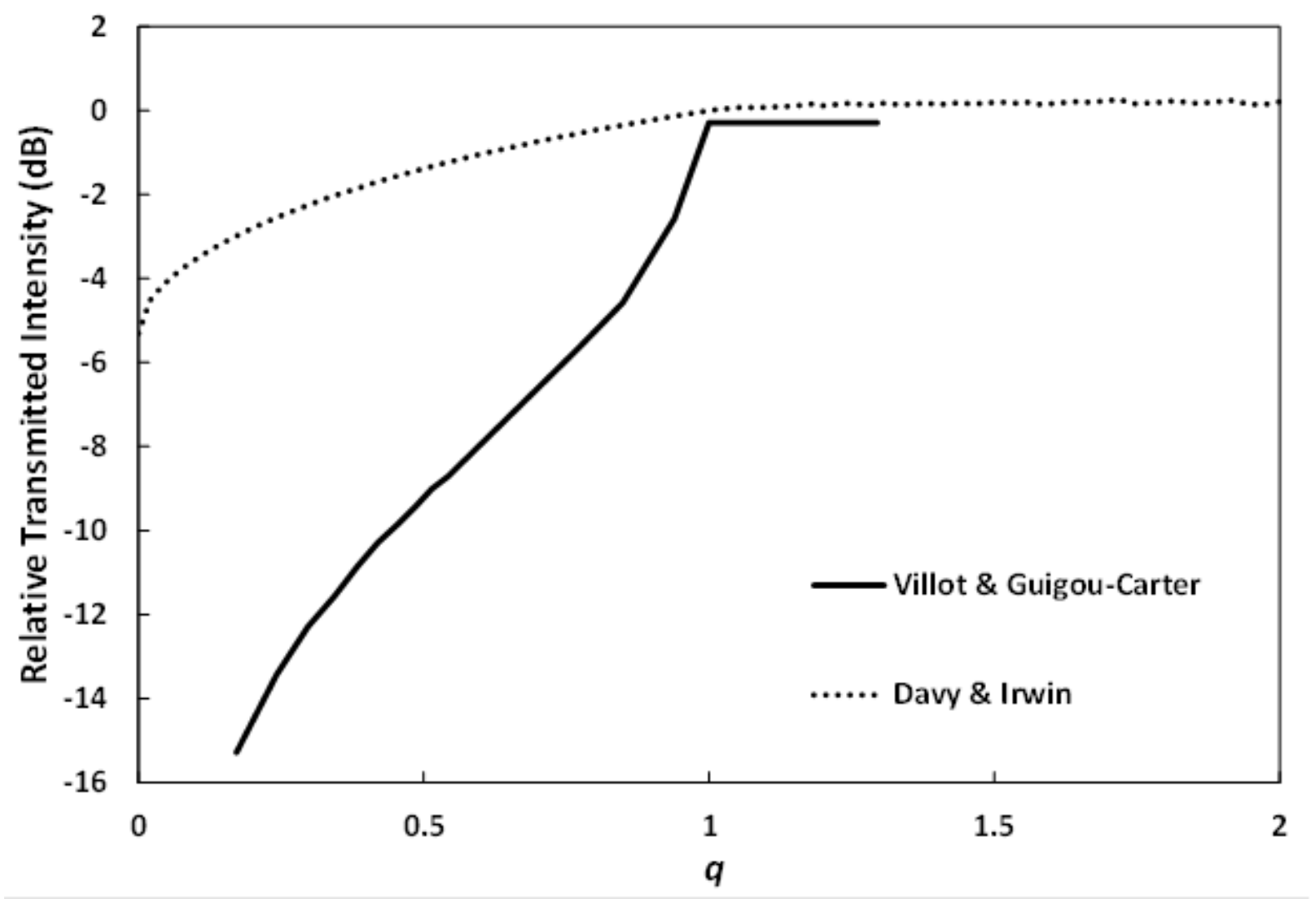

Figure 3. The relative bending wave power per unit length transmitted at a pinned joint between two panels when the two panels have the same material properties. The first panel is excited on one of its sides by a diffuse sound field. The Davy \& Irwin curve shows the calculations made in this paper, while the Villot \& Guigou-Carter curve shows the calculations made by Villot and Guigou-Carter ${ }^{2}$. The $x$-axis variable, $q$ is the ratio of the wave number of the diffuse sound field to the free bending wave number of the two identical panels. The critical frequency occurs when $q=1$. The curves are normalized so that they equal $0 \mathrm{~dB}$ when $q=1$. The mean square amplitude of the incident diffuse sound field is assumed to be constant when $q$ is varied. 